\title{
Acurácia e calibração de sonda de capacitância em Latossolo Vermelho cultivado com cafeeiro
}

\author{
Bruno Montoani Silva(1), Geraldo César de Oliveira(1), Milson Evaldo Serafim ${ }^{(2)}$, João José da Silva Júnior ${ }^{(3)}$, \\ Alberto Colombo(3) e José Maria de Lima(1) \\ (1)Universidade Federal de Lavras (Ufla), Departamento de Ciência do Solo, Caixa Postal 3037, CEP $37200-000$ Lavras, MG. \\ E-mail: montoani@posgrad.ufla.br, geraldooliveira@dcs.ufla.br, jmlima@dcs.ufla.br (2)Instituto Federal de Educação, Ciência e Tecnologia \\ de Mato Grosso, Campus Cáceres, Avenida dos Ramires, s/no, Distrito Industrial, CEP 78106-960 Cáceres, MT. \\ E-mail: milson.serafim@cas.ifmt.edu.br ${ }^{(3)}$ Ufla, Departamento de Engenharia. E-mail: jjsjunior@posgrad.ufla.br, acolombo@deg.ufla.br
}

\begin{abstract}
Resumo - O objetivo deste trabalho foi determinar a acurácia da sonda de multisensores de capacitância "Delta-T Profile probe PR2/6", na avaliação do conteúdo de água do solo com uso de calibrações padrão do fabricante, realizar a calibração para condições específicas de locais e profundidades de amostragem do solo e obter coeficientes de calibração para medições acuradas em tempo real. Em janeiro de 2010, foram coletadas amostras de solo com estrutura preservada a diferentes profundidades, nas linhas de plantio do cafeeiro e nas entrelinhas. As análises foram realizadas em laboratório, com o sensor ML2x Theta probe. Após a obtenção das leituras do sensor, o teor de água foi determinado por meio do método gravimétrico. Foram utilizadas amostras de Latossolo Vermelho distrófico muito argiloso. As calibrações padrão do fabricante (mineral e orgânica) não não se mostraram adequadas para emprego nas condições de manejo (locais e profundidades de amostragem) avaliadas. $\mathrm{Na}$ impossibilidade de averiguar a acurácia obtida pelo método recomendado pelo fabricante, o uso de ajustes de regressão linear ou da ferramenta Solver mostrou-se útil no processo de calibração. São necessárias apenas duas equações de calibração para avaliação do teor de água das situações contrastantes de manejo.
\end{abstract}

Termos para indexação: Coffea arabica, constante dielétrica, manejo do solo, solos intemperizados, sonda FDR, umidade no solo.

\section{Accuracy and calibration of capacitance probe in a Rhodic Ferralsol planted with coffee}

\begin{abstract}
The objective of this work was to determine the accuracy of the Delta-T Profile PR2/6 multisensor capacitance probe in the evaluation of soil moisture content using default calibration equations from the manufacturer, to perform the calibration for specific soil sampling locations and depths, and to obtain calibration coefficients for accurate measurements in real time. In January 2010, samples were taken with structure preserved at varying soil depths, at the planting rows or in between the rows. The analyses were done in laboratory using the ML2x Theta probe sensor. After obtaining the outputs from the sensor, water content was determined by the gravimetric method. Samples were taken from a clayed Rhodic Ferralsol. The manufacturer calibration standards (mineral and organic) were not suitable for the evaluated management (sampling location and depths) conditions. Given the impossibility to verify the accuracy obtained by the method indicated by the manufacturer, the use of linear regression adjustments or of the Solver tool was useful in the calibration process. Only two calibration equations are necessary to evaluate the contrasting management situations.
\end{abstract}

Index terms: Coffea arabica, soil dielectric constant, soil management, weathered soils, FDR probe, soil moisture.

\section{Introdução}

Conhecer o teor de água no solo é fundamental para a determinação do balanço hídrico local, do potencial de transporte de nutrientes para as plantas, da "produção" de água para mananciais hídricos e do momento mais adequado de preparo do solo, bem como para o manejo da irrigação. O método padrão para a determinação deste parâmetro é o método da estufa, e a acurácia de qualquer outro método depende da proximidade do valor obtido em relação ao método padrão. A calibração é a metodologia mais aceita para reduzir essas diferenças, e qualquer diferença não removida pela calibração é atribuída à precisão do método ou do sensor (Evett et al., 2006).

A partir de 1980, surgiram vários métodos baseados em respostas às propriedades eletromagnéticas do solo (EM) como alternativa à medição direta do teor de água do solo (ع) (Topp et al., 1980; Dean et al., 1987; Paltineanu \& Starr, 1997). Esses métodos indiretos têm sido difundidos por apresentar vantagens, como: 
resposta rápida, medições não destrutivas, precisão aceitável (Stacheder et al., 2009), além da não emissão de radiação ionizante e da possibilidade de várias medições simultâneas e do armazenamento contínuo de dados por longos períodos, o que facilita o monitoramento.

O princípio dos métodos EM é a emissão de sinal elétrico e a medição da resposta a este sinal (geralmente o tempo de reflexão ou a frequência), que ocorre em razão de mudanças na permissividade aparente ou na constante dielétrica $\left(\varepsilon_{\mathrm{a}}\right)$ do solo (Evett et al., 2006). Aágua é o componente com maior influência sobre a $\varepsilon_{\mathrm{a}} \mathrm{e}$ apresenta permissividade relativa muito maior $\left(\varepsilon_{\mathrm{a}}=81\right)$ do que a de partículas minerais e orgânicas do solo $\left(\varepsilon_{\mathrm{a}}=2-7\right)$, e do ar $\left(\varepsilon_{\mathrm{a}}=1\right)$, o que possibilita a estimativa do $\theta$ pela medição da $\varepsilon_{\mathrm{a}}$ (Topp et al., 1980).

A medição do teor de água em camadas de solo mais profundas, por meio de sensores de "time domain reflectometry" (TDR) ou por gravimetria, requer o uso de trincheiras e de numerosas amostragens ou a instalação de hastes metálicas, o que dificulta e onera o monitoramento em áreas maiores (Veldkamp \& O'Brien, 2000; Nijland et al., 2010). Assim, sondas com multisensores de capacitância (MCP) ou "frequence domain reflectometry" (FDR), que permitem a medição em várias profundidades e locais em tempo real, têm sido utilizadas no manejo da irrigação em diferentes culturas (Guerra et al., 2005), na comparação de métodos de preparo do solo (Starr \& Paltineanu, 1998), na avaliação do movimento de nutrientes (Oelmann et al., 2007), no balanço hídrico em áreas cultivadas (Fares et al., 2006; Prevedello et al., 2007) e no monitoramento da umidade do perfil do solo em ecossistemas naturais e áreas de cultivo (Nijland et al., 2010). O princípio de funcionamento dos MCP se baseia na redução da frequência de ressonância à medida que o teor de água (permissividade) do meio aumenta (Evett et al., 2006).

Os sensores MCP e TDR apresentam equações de calibração padrão de fábrica que relacionam o teor de água à leitura de saída do sensor. Entretanto, para medições mais acuradas, é necessário realizar calibrações para os diferentes solos, em virtude das propriedades eletromagnéticas inerentes à cada classe de solo (Fares et al., 2006; Prevedello et al., 2007). Calibrações têm sido realizadas em condições de campo (Polyakov et al., 2005; Prevedello et al., 2007; Silva et al., 2007) e em laboratório (Paltineanu \& Starr,
1997; Baumhardt et al., 2000; Veldkamp \& O'Brien, 2000; Fares et al., 2006; Lukanu \& Savage, 2006). Observou-se que a acurácia dos modelos encontrada em condições de campo tem sido inferior à obtida em laboratório (Paltineanu \& Starr, 1997; Fares et al., 2006), o que é atribuído ao pequeno volume de solo abrangido na medição das sondas, às variações de densidade do solo, ao próprio teor de água junto ao tubo de acesso e a efeitos de deformações ocorridas durante a instalação do tubo de acesso (Evett et al., 2006).

O número de estudos com calibração em solos de clima tropical é limitado (Veldkamp \& O'Brien, 2000; Polyakov et al., 2005; Fares et al., 2006), e é menor ainda em solos do bioma Cerrado brasileiro (Sá et al., 2008). Em Latossolos argilosos, em razão de algumas particularidades, como os altos teores de óxidos e hidróxidos de ferro, a calibração específica é indispensável (Villwock, 2004). Silva \& Gervásio (1999) obtiveram valores subestimados do teor de água medido por sonda TDR em um Latossolo muito argiloso, ao utilizar a calibração padrão do fabricante.

Evett et al. (2006) propuseram que a calibração dos MCP seja separada por horizonte do solo, uma vez que a permissividade aparente é influenciada por teores de matéria orgânica e argila (Veldkamp \& O'Brien, 2000; Sá et al., 2008; Kaiser et al., 2010), óxidos e hidróxidos de ferro (Prevedello et al., 2007; Kaiser et al., 2010), condutividade elétrica, temperatura (Tomaselli \& Bacchi, 2001; Evett et al., 2006) e densidade do solo (Malicki et al., 1996; Huang et al., 2004); atributos que podem apresentar variação no perfil do solo.

O objetivo deste trabalho foi determinar a acurácia da sonda de multisensores de capacitância "Delta-T Profile probe PR2/6", na avaliação do conteúdo de água do solo com uso de calibrações padrão do fabricante, realizar a calibração para condições específicas de locais e profundidades de amostragem do solo e obter coeficientes de calibração para medições acuradas em tempo real.

\section{Material e Métodos}

O solo avaliado foi um Latossolo Vermelho distrófico muito argiloso, cultivado com cafeeiro (Coffea arabica L.), na região de São Roque de Minas, MG $\left(20^{\circ} 15^{\prime} \mathrm{S}\right.$ e $46^{\circ} 22^{\prime} \mathrm{W}$, a $900 \mathrm{~m}$ de altitude). O clima da região, conforme a classificação de Köppen, é do tipo Cwa. 
$\mathrm{Na}$ fase de implantação do sistema de manejo do solo, foi feito revolvimento profundo na linha de plantio do cafeeiro, tendo-se deixado sulco com $60 \mathrm{~cm}$ de profundidade e $50 \mathrm{~cm}$ de largura, o que causou alterações na estrutura original do solo. $\mathrm{Na}$ entrelinha do cafeeiro, foi implantada cobertura permanente de capim braquiária (Urochloa sp.), manejada com cortes periódicos. Após implantação do cafeeiro, com plantas com altura superior a $50 \mathrm{~cm}$, adicionouse $7,0 \mathrm{~kg} \mathrm{~m}^{-1}$ de gesso agrícola na linha de plantio. Em seguida, foi removida a camada superficial da entrelinha e realizada amontoa na linha do cafeeiro. Foi determinada a granulometria de amostras do solo, para a sua caracterização (Tabela 1). Para identificar as diferenças nas camadas de solo resultantes desse manejo, que provavelmente influenciaram a permissividade dielétrica aparente do meio, foram analisados a densidade do solo, o teor de matéria orgânica e a capacidade de troca do complexo sortivo, para as profundidades de: $0-0,1$ e $0,2-0,3 \mathrm{~m}$, na linha de plantio do cafeeiro (L); $0-0,1$ e $0,1-0,2 \mathrm{~m}$, na entrelinha (E); e $0,75-0,85 \mathrm{~m}$, no horizonte Bw (Tabela 2).

Em janeiro de 2010, amostras com estrutura preservada foram coletadas com anéis cilíndricos de PVC rígido, com $10 \mathrm{~cm}$ de diâmetro e $10 \mathrm{~cm}$ de altura, em três repetições, para cada camada de solo avaliada, para se obter a quantidade de solo suficientemente representativa para a inserção do sensor de umidade. O sensor de capacitância utilizado para medição do teor de água, modelo ML2x Theta probe (Delta-T
Devices Ltd., Cambridge, Reino Unido), tem quatro hastes metálicas, que geram um sinal de $100 \mathrm{MHz}$. Como saída, recebe um valor de voltagem (V) que é determinado por sua relação com a raiz quadrada da permissividade dielétrica aparente $\left(\varepsilon_{\mathrm{a}}{ }^{0,5}\right.$ ou índice refrativo), por meio de equação polinomial intrínseca do sensor, denominada de resposta do sensor (equação 1) (Delta-T Devices, 1999). A conversão do valor de saída do sensor em teor de água do solo se dá por meio da relação existente entre o índice refrativo e o teor de água do meio, conhecida como calibração do solo (equação 2). A equação de calibração do solo varia de solo para solo, em função de suas particularidades, e pode ser expressa por equação linear, em que os coeficientes de ajuste $a_{0}$ e $a_{1}$ representam as particularidades. Os coeficientes obtidos podem ser inseridos no leitor de mão usado em campo, para medições mais acuradas em tempo real.

São duas as calibrações do solo consideradas como padrão de fábrica: a "mineral" $\left(\mathrm{a}_{0}=1,6 \mathrm{e}\right.$ $\left.\mathrm{a}_{1}=8,4\right)$, indicada para solos com teor de matéria orgânica inferior a 7\% e Ds $>1,0 \mathrm{~g} \mathrm{dm}^{-3}$; e a "orgânica" $\left(a_{0}=1,3\right.$ e $\left.a_{1}=7,7\right)$, indicada para teores de matéria orgânica maiores que $7 \%$ e Ds $<1,0 \mathrm{~g} \mathrm{dm}^{-3}$. O sensor ML2x apresenta acurácia de $\pm 0,05 \mathrm{~m}^{3} \mathrm{~m}^{-3}\left(0-70^{\circ} \mathrm{C}\right)$, com uso de calibrações de fábrica, e de $\pm 0,01 \mathrm{~m}^{3} \mathrm{~m}^{-3}$, com calibração específica para o solo no qual se vai trabalhar, sem erros significativos para solos de 250 a $2.000 \mathrm{mS} \mathrm{m}^{-1}$ de condutividade elétrica. A diferença no

Tabela 1. Granulometria e resultados do ataque sulfúrico de amostras de horizontes diagnósticos superficiais e subsuperficiais de Latossolo Vermelho distrófico.

\begin{tabular}{lccccccccccc}
\hline Horizonte & $\begin{array}{c}\text { Profundidade } \\
(\mathrm{m})\end{array}$ & Areia & Silte & Argila & $\mathrm{SiO}_{2}$ & $\mathrm{Al}_{2} \mathrm{O}_{3}$ & $\mathrm{Fe}_{2} \mathrm{O}_{3}$ & $\mathrm{KiO}_{3}$ & $\mathrm{Ki}$ \\
\hline $\mathrm{A}$ & $0-0,2$ & 50 & 180 & 770 & 102 & 355 & 157 & 13,2 \\
$\mathrm{Bw}$ & $0,6-0,8$ & 40 & 160 & 790 & 105 & 392 & 169 & 12,7 & 0,49 & 0,46 & 0,36 \\
\hline
\end{tabular}

Tabela 2. Análises química e física para as diferentes camadas e locais de amostragem.

\begin{tabular}{|c|c|c|c|c|c|c|c|c|c|c|c|c|}
\hline Posição & $\begin{array}{c}\text { Camada } \\
(\mathrm{m})\end{array}$ & $\begin{array}{c}\mathrm{pH} \mathrm{em} \\
\mathrm{H}_{2} \mathrm{O}\end{array}$ & SB & $\begin{array}{l}\mathrm{H}+\mathrm{Al} \\
--(\mathrm{cmo}\end{array}$ & $\begin{array}{c}\mathrm{t} \\
\left.\mathrm{m}^{-3}\right)\end{array}$ & $\mathrm{T}$ & V & m & $\begin{array}{c}\mathrm{CE} \\
\left(\mathrm{mS} \mathrm{m}^{-1}\right)\end{array}$ & $\begin{array}{c}\mathrm{MO} \\
\left(\mathrm{dag} \mathrm{kg}^{-1}\right)\end{array}$ & $\begin{array}{c}\text { Ds } \\
\left(\mathrm{kg} \mathrm{m}^{-3}\right)\end{array}$ & $\begin{array}{c}\text { VTP } \\
\left(\mathrm{m}^{3} \mathrm{~m}^{-3}\right)\end{array}$ \\
\hline \multirow{2}{*}{ Linha } & $0-0,1$ & 5,6 & 5,3 & 4,0 & 5,4 & 9,3 & 56,5 & 1,9 & 60 & 4,1 & 0,86 & 0,68 \\
\hline & $0,2-0,3$ & 6,0 & 6,2 & 3,2 & 6,2 & 9,4 & 65,7 & 0,0 & 80 & 3,4 & 0,85 & 0,68 \\
\hline \multirow{2}{*}{ Entrelinha } & $0-0,1$ & 5,4 & 3,5 & 5,6 & 3,7 & 9,1 & 38,1 & 5,5 & 31 & 4,0 & 0,95 & 0,64 \\
\hline & $0,1-0,2$ & 4,6 & 0,9 & 8,8 & 1,7 & 9,7 & 9,4 & 46,8 & 24 & 3,1 & 0,91 & 0,66 \\
\hline Horizonte $\mathrm{Bw}$ & $0,75-0,85$ & 5,3 & 1,4 & 4,0 & 1,5 & 5,5 & 26,0 & 6,6 & 16 & 1,6 & 0,65 & 0,65 \\
\hline
\end{tabular}

SB, soma de bases; t, capacidade efetiva de troca de cátions; T, capacidade potencial de troca de cátions; V, saturação por bases; m, saturação por alumínio; CE, condutividade elétrica; MO, matéria orgânica do solo; Ds, densidade do solo; VTP, volume total de poros. 
processo de obtenção do teor de água da sonda ML2x para a sonda de multisensores de capacitância, Profile probe PR2/6 (Delta-T Devices Ltd., Cambridge, Reino Unido), é representada pela equação de resposta (equação 3). Assim, ao se realizar leituras com essas sondas, o valor de saída do sensor (V) é diferente, mas quando este valor é inserido na equação de resposta da respectiva sonda (equações 1 e 3 ), espera-se um mesmo valor de $\varepsilon^{0,5}$. Ao se determinar os valores de $\varepsilon^{0,5}$ e teor de água do solo $(\theta)$, é possível obter os coeficientes de calibração $\mathrm{a}_{0}$ e $\mathrm{a}_{1}$ com uso da sonda ML2x e usá-los para a PR2/6, conforme o manual do fabricante (Delta-T Devices, 2001). Desse modo, os dados utilizados foram obtidos por meio da ML2x. Foram utilizadas as equações,

$\varepsilon^{0,5}=1,07+6,4 \mathrm{~V}-6,4 \mathrm{~V}^{2}+4,7 \mathrm{~V}^{3}$

$\varepsilon^{0,5}=a_{0}+a_{1} \times \theta$

$\varepsilon^{0,5}=1,125-5,53 \mathrm{~V}+67,17 \mathrm{~V}^{2}-234,42 \mathrm{~V}^{3}+$

$413,56 \mathrm{~V}^{4}-356,68 \mathrm{~V}^{5}+121,53 \mathrm{~V}^{6}$

em que: $\varepsilon^{0,5}$ é a raiz quadrada da permissividade dielétrica; $\mathrm{a}_{0}$ e $\mathrm{a}_{1}$ são os parâmetros de calibração, específicos para cada solo; $\theta$ representa o teor de água volumétrico, em $\mathrm{m}^{3} \mathrm{~m}^{-3}$; e $\mathrm{V}$ representa a leitura de saída do sensor, em volts.

Determinou-se o peso inicial (Pi) das amostras, que então foram colocadas em estufa a $60^{\circ} \mathrm{C}$, até atingirem $\theta$ de 25 a $30 \%$. Em seguida, a sonda ML2x foi inserida em cada amostra, para obter-se a leitura de saída. Novamente, as amostras foram secas em estufa a $60^{\circ} \mathrm{C}$, até terem $\theta$ próximo de $15 \%$, quando foi realizada outra leitura com a ML2x. Posteriormente, as amostras foram secas em estufa a $105^{\circ} \mathrm{C}$ para determinação do peso seco. Ao final, dois pares de valores (saída do sensor e $\theta$ ) foram obtidos, de acordo com o recomendado pelo manual do fabricante (Delta-T Devices, 2001). Para estimar o peso com que as amostras estariam com o $\theta$ próximo do valor desejado (entre $25 \mathrm{e}$ $30 \%$, e $15 \%$ ), foi realizada uma repetição extra, para determinar o peso seco e a densidade do solo, o que permitiu estimar o peso úmido desejado para as demais amostras, conforme as etapas a seguir: obtenção do peso inicial e do peso seco (estufa a $105^{\circ} \mathrm{C}$ ); cálculo do teor de água inicial $(\Theta)$, da densidade do solo (Ds = peso seco por volume) e da umidade gravimétrica $\left(\mathrm{U}, \mathrm{g} \mathrm{g}^{-1}\right)$, por meio da relação $\Theta=\mathrm{U}$.Ds; estimação do peso seco de cada amostra, por meio da relação $\mathrm{Pu}=\mathrm{Ps}(1+\mathrm{U})$, sendo os pesos iniciais mensurados e considerados como $\mathrm{Pu}$; estimação do peso úmido na umidade desejada $(\Theta)$, pela umidade correspondente (U) e pelo peso seco estimado anteriormente, por meio da relação $\mathrm{Pu}=\operatorname{Ps}(1+\mathrm{U})$.

A acurácia das calibrações de fábrica foi avaliada por meio da raiz do erro-médio quadrático (RMSE) e da reta 1:1 entre os valores observados ( $\theta$ obtido por gravimetria) e os estimados ( $\theta$ obtido pelas calibrações padrão de fábrica, com uso da equação de resposta da ML2x).

Em seguida, procedeu-se à calibração para cada camada de solo, por três processos. No primeiro, com os dois pares de valores, $\theta$ e $\varepsilon^{0,5}$ (obtidos pela equação de resposta da ML2x), para $\theta$ próximos de 25 e $15 \%$, montou-se um sistema de equações com a equação de calibração (equação 2), tendo-se obtido os coeficientes $\mathrm{a}_{0}$ e $\mathrm{a}_{1}$, conforme recomendado pelo fabricante (Delta-T Devices, 2001). Uma vez que o processo não prevê o uso de repetições, o que gera incertezas sobre os coeficientes obtidos, em virtude das variações entre amostras de mesmo solo, os modelos lineares foram ajustados entre $\varepsilon^{0,5}$ e $\theta$ (obtido por gravimetria), para toda a série de dados e para cada camada de solo. Além das regressões lineares, foi calculado o quadrado da soma dos desvios, que foi minimizado por meio da ferramenta Solver do Microsoft Excel, para os valores observados e estimados, tendo-se obtido os coeficientes lineares e angulares.

A acurácia dos modelos de calibração foi avaliada por meio do coeficiente de determinação $\left(\mathrm{R}^{2}\right)$, com uso da RMSE, e por meio da reta 1:1 entre os valores observados e os estimados. A validação do modelo foi realizada pelo índice de concordância (d) de Willmott (1981) e pelo índice de confiança (c) de Camargo \& Sentelhas (1997). Para os indicadores $\mathrm{R}^{2}$, $\mathrm{d}$ e c (0 a 1), quanto mais próximo de 1 , melhor o ajuste dos modelos, e, para RMSE, valores próximos de zero são desejados. Para a definição das equações de calibração a serem utilizadas, foi construído o intervalo de confiança (IC) e determinado o erro-padrão para o coeficiente angular e o intercepto das retas ajustadas, o que permitiu verificar semelhanças e diferenças entre os modelos por camada e para toda a série de dados, tendo-se selecionado apenas os que diferiram entre si. 


\section{Resultados e Discussão}

O teor de água medido pela sonda de capacitância, com uso de calibração mineral, para todos os locais avaliados, foi subestimado em comparação ao método padrão da estufa, em toda a faixa de umidade avaliada, como indica a posição da reta ajustada entre os valores observados e os valores medidos na calibração mineral, sempre abaixo da reta 1:1 (Figura 1). Esse resultado pode ser atribuído aos altos teores de óxidos de ferro e ao elevado grau de intemperização do solo analisado (Tabelas 1 e 2), que afetam a constante dielétrica e, consequentemente, a umidade estimada (Villwock et al., 2004; Santos et al., 2010) em virtude da presença elevada de cargas positivas no complexo de troca. Vários autores relataram valores subestimados para o teor de água do solo com uso da equação de Topp et al. (1980), que relaciona o teor de água à constante dielétrica, considerada como equação padrão em equipamentos TDR (Silva \& Gervásio, 1999; Santos et al., 2010). Isso indica que as calibrações de fábrica dos MCP não atendem ao propósito da universalização, assim como a equação de Topp et al. (1980) para sondas TDR (Tommaselli \& Bacchi, 2001), o que pode ser decorrente das particularidades dos solos tropicais, principalmente latossolos, e da necessidade de calibração específica para estes solos.

Na calibração orgânica de fábrica, o teor de água observado foi subestimado a partir de valores próximos de 0,10 a $0,15 \mathrm{~m}^{3} \mathrm{~m}^{-3}$ e superestimado para valores de umidade menores, para todas as camadas avaliadas (Figura 1). De acordo com o manual do fabricante, essa calibração é indicada para solos com teores de matéria orgânica superiores a $7 \%$ e Ds $<1,0 \mathrm{~g} \mathrm{dm}^{-3}$, mas não há referência à textura e à mineralogia. Apesar do comportamento semelhante das equações, observado para cada camada de solo (Tabelas 1 e 2), não foi possível isolar os fatores que condicionaram esse comportamento.

Kaiser et al. (2010), ao avaliar materiais de Latossolo Vermelho distrófico com 549 dag $\mathrm{kg}^{-1}$ de argila e ki de 2,12, verificaram que o modelo de Topp et al. (1980) subestimou a umidade observada, nos menores valores, e a superestimou nos maiores valores, o que foi atribuído ao alto grau de intemperismo e aos teores elevados de óxido de ferro. Assim, ao se considerar que o solo analisado é mais intemperizado (ki de 0,46 ) e apresenta teores consideráveis de $\mathrm{Fe}_{2} \mathrm{O}_{3}$ (Tabela 1), espera-se comportamento diferente do Latossolo avaliado por Kaiser et al. (2010).
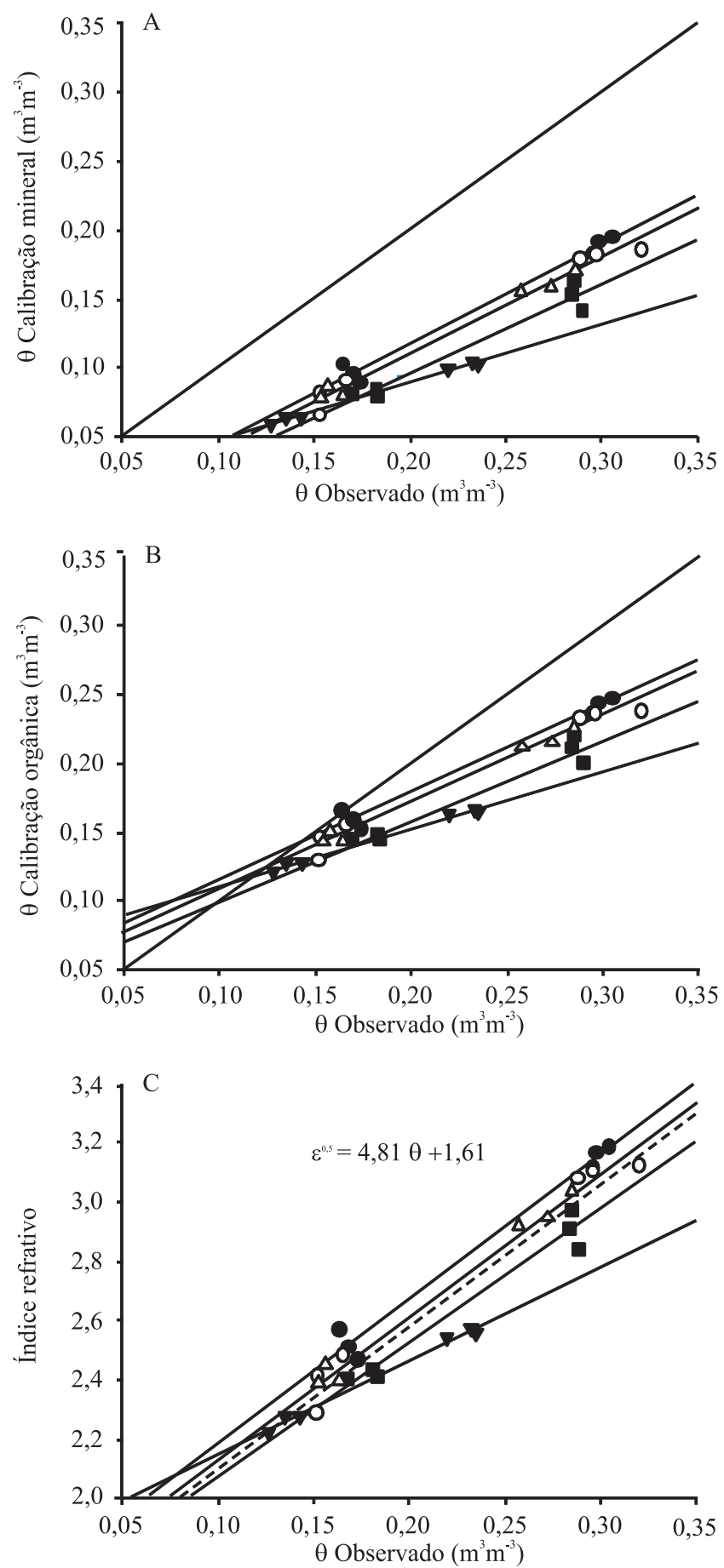

$\nabla \mathrm{L} 0,0-0,1 \mathrm{~m} \quad \Delta \mathrm{L} 0,2 \quad 0,3 \mathrm{~m} \quad \bullet \mathrm{E} 0,0-0,1 \mathrm{~m}$ ○ E $0,1-0,2 \mathrm{~m}$ - Bw $0,75-0,1 \mathrm{~m}$ - - Série completa

Figura 1. Relação entre o teor de água determinado por gravimetria (método padrão) e por sonda, com uso de: A, calibração de fábrica "mineral"; B, calibração de fábrica "orgânica"; C, ajuste de modelo de regressão linear entre o índice refrativo $\left(\varepsilon^{0,5}\right)$ e o teor de água do solo. 
A calibração mineral apresentou maior erro ou menor acurácia na determinação da umidade, em comparação à orgânica (Tabela 3). As maiores diferenças foram observadas nas camadas $\mathrm{Bw}(0,75-0,85 \mathrm{~m})$ e L (0-0,10 m). A RMSE indicou que, em média, os desvios foram maiores na calibração mineral do que na orgânica, tendo-se verificado, na camada $\mathrm{Bw}(0,75-$ $0,85 \mathrm{~m})$, valores acima do erro tolerado $\left( \pm 0,05 \mathrm{~m}^{3} \mathrm{~m}^{-3}\right)$ pelo manual do fabricante. Cabe ressaltar, no entanto, que os valores de RMSE tolerados não excluem a necessidade de calibração específica para cada camada de solo, pois há desvios consideráveis em relação à reta 1:1 para ambas as calibrações de fábrica (Figura 1).

Todas as camadas de solo avaliadas apresentaram teores de matéria orgânica inferiores a $7 \%$ e Ds $<1,0 \mathrm{~g} \mathrm{dm}^{-3}$; portanto, não se adequaram a nenhuma das duas calibrações de fábrica (Tabela 2). Assim, ao se considerar os desvios em relação à umidade observada (Figura 1), recomenda-se calibração específica para cada camada de solo.

$\mathrm{Na}$ metodologia descrita pelo fabricante, não há previsão de uso de repetições para obtenção dos coeficientes de calibração. No entanto, no presente trabalho, foram feitas três repetições de cada camada, tendo-se obtido diferentes coeficientes para a mesma situação (Tabela 4). Quanto às médias, foram

Tabela 3. Acurácia das calibrações mineral e orgânica, padrões de fábrica.

\begin{tabular}{lcccccc}
\hline Camada de solo & Gravimétrica $^{(1)}$ & \multicolumn{2}{c}{ Mineral } & & \multicolumn{2}{c}{ Orgânica } \\
\cline { 3 - 4 } \cline { 7 - 8 } & & Erro $^{(2)}$ & RMSE $^{(3)}$ & & Erro $^{(2)}$ & RMSE $^{(3)}$ \\
\hline $0-0,10 \mathrm{~m}$, linha & 0,230 & 0,128 & 0,104 & & 0,066 & 0,048 \\
$0,20-0,30 \mathrm{~m}$, linha & 0,273 & 0,111 & 0,096 & & 0,055 & 0,041 \\
$0-0,10 \mathrm{~m}$, entrelinha & 0,300 & 0,110 & 0,094 & & 0,059 & 0,043 \\
$0,10-0,20 \mathrm{~m}$, entrelinha & 0,302 & 0,120 & 0,102 & & 0,068 & 0,050 \\
0,75-0,85 m, horizonte Bw & 0,287 & 0,134 & 0,117 & & 0,077 & 0,060 \\
\hline
\end{tabular}

(1)Valores médios de umidade volumétrica para o ponto mais úmido avaliado. ${ }^{(2)}$ Diferença dos valores observados em comparação aos determinados pelas equações de fábrica. ${ }^{(3)}$ Raiz do erro médio quadrático para toda a série de dados obtida pelas calibrações de fábrica, em comparação aos valores observados. observadas diferenças entre os coeficientes obtidos em cada camada, principalmente por meio da análise dos coeficientes obtidos por regressão e minimização dos erros (Solver).

Os ajustes lineares entre o teor de água observado e a raiz quadrada da constante dielétrica (índice refrativo) foram significativos $(p<0,01)$ em todas as camadas de solo e no ajuste de toda a série de dados (Figura 1). Outros modelos de ajuste que relacionam a constante dielétrica ao teor de água, como o polinomial e o potencial, podem resultar em maiores coeficientes de determinação, como observado em outros trabalhos (Coelho et al., 2001; Tommaselli \& Bacchi, 2001; Kaiser et al., 2010). Contudo, há vantagem em se utilizar modelos lineares, como o de calibração do solo para MCP Profile probe PR2, que é a medição em tempo real da umidade no campo. Isso é possível porque há uma opção no leitor do equipamento que permite inserir os coeficientes de reta $a_{0}$ e $a_{1}$ (ajuste linear), o que elimina a necessidade de corrigir as leituras obtidas em campo. A possibilidade do uso de equações lineares para a calibração de solos com altos teores de argila, a exemplo do Latossolo avaliado, foi observada por Santos et al. (2010), que analisaram solos com teores crescentes de argila e verificaram tendência de linearização da equação de calibração à medida que se elevavam os teores dessa fração granulométrica no solo.

Em todas as avaliações, para todas as camadas e localizações da amostragem, independentemente do método de obtenção dos coeficientes, foram encontrados altos índices de Wilmott $(>0,99)$, de confiança $(>0,95)$ e de determinação $(>0,96)$. O maior valor de RMSE obtido foi de $0,011 \mathrm{~m}^{3} \mathrm{~m}^{-3}$, para a camada $\mathrm{Bw}(0,75-$ $0,85 \mathrm{~m}$ ). Esses índices são indicativos da confiabilidade da acurácia dos modelos, para todas as condições avaliadas. Os modelos que utilizam toda a série de dados apresentaram menores valores para os índices de acurácia, em comparação aos modelos individuais

Tabela 4. Coeficientes de calibração $\left(\mathrm{a}_{0} \text { e } \mathrm{a}_{1}\right)^{(1)}$ para cada camada de solo, conforme recomendado pelo manual do fabricante.

\begin{tabular}{|c|c|c|c|c|c|c|c|c|c|c|}
\hline \multirow[t]{2}{*}{ Repetições } & \multicolumn{2}{|c|}{$0-0,10 \mathrm{~m}$ linha } & \multicolumn{2}{|c|}{$0,20-0,30 \mathrm{~m}$ linha } & \multicolumn{2}{|c|}{$0-0,10 \mathrm{~m}$ entrelinha } & \multicolumn{2}{|c|}{$0,10-0,20 \mathrm{~m}$ entrelinha } & \multicolumn{2}{|c|}{$0,75-0,85 \mathrm{~m}$ horizonte $\mathrm{Bw}$} \\
\hline & $\mathrm{a}_{0}$ & $\mathrm{a}_{1}$ & $\mathrm{a}_{0}$ & $\mathrm{a}_{1}$ & $\mathrm{a}_{0}$ & $\mathrm{a}_{1}$ & $\mathrm{a}_{0}$ & $a_{1}$ & $\mathrm{a}_{0}$ & $\mathrm{a}_{1}$ \\
\hline 1 & 1,860 & 3,072 & 1,603 & 5,129 & 1,837 & 4,450 & 1,412 & 5,777 & 1,375 & 5,617 \\
\hline 2 & 1,797 & 3,375 & 1,522 & 5,313 & 1,693 & 4,811 & 1,786 & 4,175 & 1,664 & 4,397 \\
\hline 3 & 1,831 & 3,106 & 1,780 & 4,268 & 1,508 & 5,517 & 1,677 & 4,816 & 1,749 & 3,765 \\
\hline Média & 1,829 & 3,184 & 1,635 & 4,903 & 1,679 & 4,926 & 1,625 & 4,923 & 1,596 & 4,593 \\
\hline
\end{tabular}

${ }^{(1)} \mathrm{a}_{0}$, coeficiente linear ou intercepto; $\mathrm{a}_{1}$, coeficiente angular. 
por camada, com destaque para o índice de confiança $(0,906)$ e a RMSE $(0,018)$.

Ao se comparar as duas metodologias (Tabela 5), observaram-se diferenças nos valores dos coeficientes de ajuste por camada avaliada, principalmente quanto ao ajuste de toda a série de dados $\left(\mathrm{a}_{0}=1,618 \mathrm{e}\right.$ $\mathrm{a}_{1}=4,808$, pela regressão linear, e $\mathrm{a}_{0}=1,532$ e $\mathrm{a}_{1}=5,200$, pela ferramenta Solver). Porém, essas diferenças não resultaram em $\mathrm{R}^{2}$, d, c e RMSE diferentes entre as metodologias.

O ajuste dos valores estimados após a calibração e os valores de teor de água mostra grande coincidência com a reta 1:1 (Figura 2), o que está em concordância com os indicadores de validação das equações de calibração obtidas por regressão linear e pelo aplicativo Solver. Assim, os procedimentos de calibração adotados apresentaram ganhos importantes de acurácia na medição correta do teor de água. Esses ganhos foram visualizados por meio da diminuição da RMSE calculada antes (Tabela 3) e depois (Tabela 5) do processo, e justificam a realização da calibração. Esses resultados são indicativos de que ambos os procedimentos podem ser adotados para a calibração específica da sonda de MCP.

Ao se analisar o intervalo de confiança e o erro-padrão dos coeficientes obtidos por meio de regressão linear, para cada camada de solo e para a série toda (Figura 3), verifica-se similaridade entre os ajustes, para as camadas $\mathrm{L}(0,2-0,3 \mathrm{~m})$, Bw $(0,75-0,85 \mathrm{~m}), \mathrm{E}(0-0,1 \mathrm{~m}), \mathrm{E}(0,1-0,2 \mathrm{~m})$ e para a série toda, o que indica que qualquer uma das equações ajustadas para essas camadas pode representar as demais. Portanto, não é necessário realizar calibração específica para cada uma dessas situações, pois apenas uma equação pode ser utilizada para todas elas. O mesmo não foi observado para $\mathrm{L}(0-0,1 \mathrm{~m})$, uma vez que não houve coincidência do intervalo de confiança do coeficiente angular dessa camada com as demais, o que indica que os coeficientes são diferentes. Quanto ao intercepto, houve diferença para a camada $\mathrm{L}(0,2-0,3 \mathrm{~m})$ e para a série toda.

A exigência de calibração específica para L $(0-0,1 \mathrm{~m})$ pode ser resultante do alto teor de matéria orgânica, em comparação aos demais locais de amostragem, à exceção de $\mathrm{E}(0-0,1 \mathrm{~m})$, que apresentou o mesmo teor de matéria orgânica, porém maior densidade do solo (Tabela 2).

Maiores teores de matéria orgânica, para um mesmo teor de água do solo, resultam em menos poros preenchidos por água livre, uma vez que a água estaria adsorvida à matéria orgânica. Como a água adsorvida apresenta menor constante dielétrica aparente, a constante dielétrica é menor para um mesmo teor de água. Esse efeito provavelmente é similar ao que ocorre nas argilas 2:1, quando comparadas às argilas 1:1 (Polyakov et al., 2005), e foi atribuído à maior superfície específica das argilas 2:1.

A maior densidade do solo geralmente representa menor quantidade de macroporos, o que diminui o espaço preenchido por ar. Portanto, para um mesmo

Tabela 5. Acurácia e validação dos modelos de calibração do solo.

\begin{tabular}{|c|c|c|c|c|c|c|}
\hline \multirow[t]{2}{*}{ Camada de solo } & \multicolumn{2}{|c|}{ Coeficientes da equação de calibração } & \multirow[t]{2}{*}{$\mathrm{R}^{2}$} & \multirow[t]{2}{*}{$\mathrm{RMSE}^{(1)}$} & \multirow[t]{2}{*}{$\mathrm{d}^{(2)}$} & \multirow[t]{2}{*}{$\mathrm{c}^{(3)}$} \\
\hline & $\mathrm{a}_{0}$ (linear) & $\mathrm{a}_{1}$ (angular) & & & & \\
\hline & \multicolumn{6}{|c|}{ Regressão linear } \\
\hline Série completa & 1,618 & 4,808 & 0,925 & 0,018 & 0,980 & 0,906 \\
\hline $0-0,10 \mathrm{~m}$, linha & 1,831 & 3,169 & 0,994 & 0,004 & 0,999 & 0,993 \\
\hline $0,20-0,30 \mathrm{~m}$, linha & 1,644 & 4,865 & 0,988 & 0,006 & 0,997 & 0,985 \\
\hline $0-0,10 \mathrm{~m}$, entrelinha & 1,687 & 4,901 & 0,981 & 0,009 & 0,995 & 0,977 \\
\hline $0,10-0,20 \mathrm{~m}$, entrelinha & 1,637 & 4,847 & 0,978 & 0,011 & 0,994 & 0,973 \\
\hline \multirow[t]{2}{*}{ 0,75-0,85 m, horizonte $\mathrm{Bw}$} & 1,612 & 4,524 & 0,960 & 0,011 & 0,990 & 0,950 \\
\hline & \multicolumn{6}{|c|}{ Solver (minimização do MSE) } \\
\hline Série completa & 1,532 & 5,200 & 0,925 & 0,017 & 0,980 & 0,906 \\
\hline $0-0,10 \mathrm{~m}$, linha & 1,828 & 3,188 & 0,994 & 0,004 & 0,999 & 0,993 \\
\hline $0,20-0,30 \mathrm{~m}$, linha & 1,631 & 4,923 & 0,988 & 0,006 & 0,997 & 0,985 \\
\hline $0-0,10 \mathrm{~m}$, entrelinha & 1,665 & 4,994 & 0,981 & 0,009 & 0,995 & 0,977 \\
\hline $0,10-0,20 \mathrm{~m}$, entrelinha & 1,612 & 4,955 & 0,978 & 0,011 & 0,994 & 0,973 \\
\hline 0,75-0,85 m, horizonte $\mathrm{Bw}$ & 1,568 & 4,713 & 0,960 & 0,011 & 0,990 & 0,950 \\
\hline
\end{tabular}

(1)Raiz do erro-médio quadrático entre os valores observados e os estimados por meio das equações de calibração do solo, para cada situação de manejo e para toda a série de dados. ${ }^{(2)}$ Índice de Willmott. ${ }^{(3)}$ Índice de confiança. 

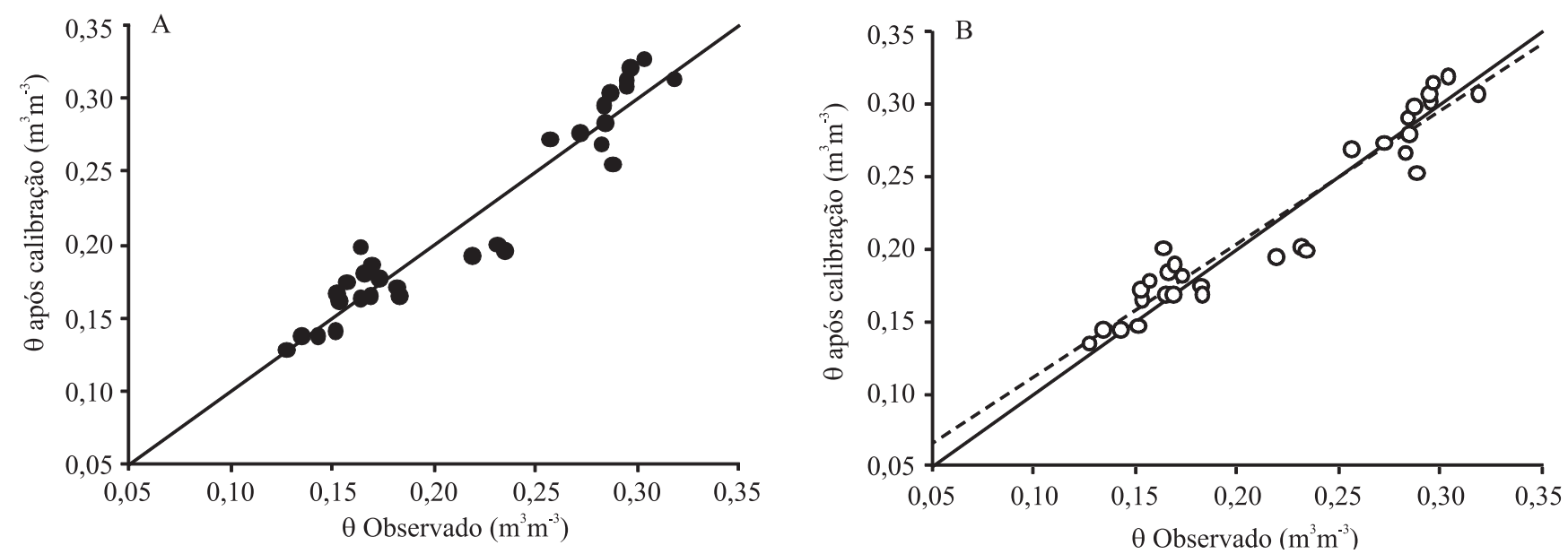

Figura 2. Retas 1:1 entre o teor de água volumétrico observado (estufa) e o estimado por meio de calibrações ajustadas para toda a série de dados. A, regressão linear (série completa); B, Solver (série completa).

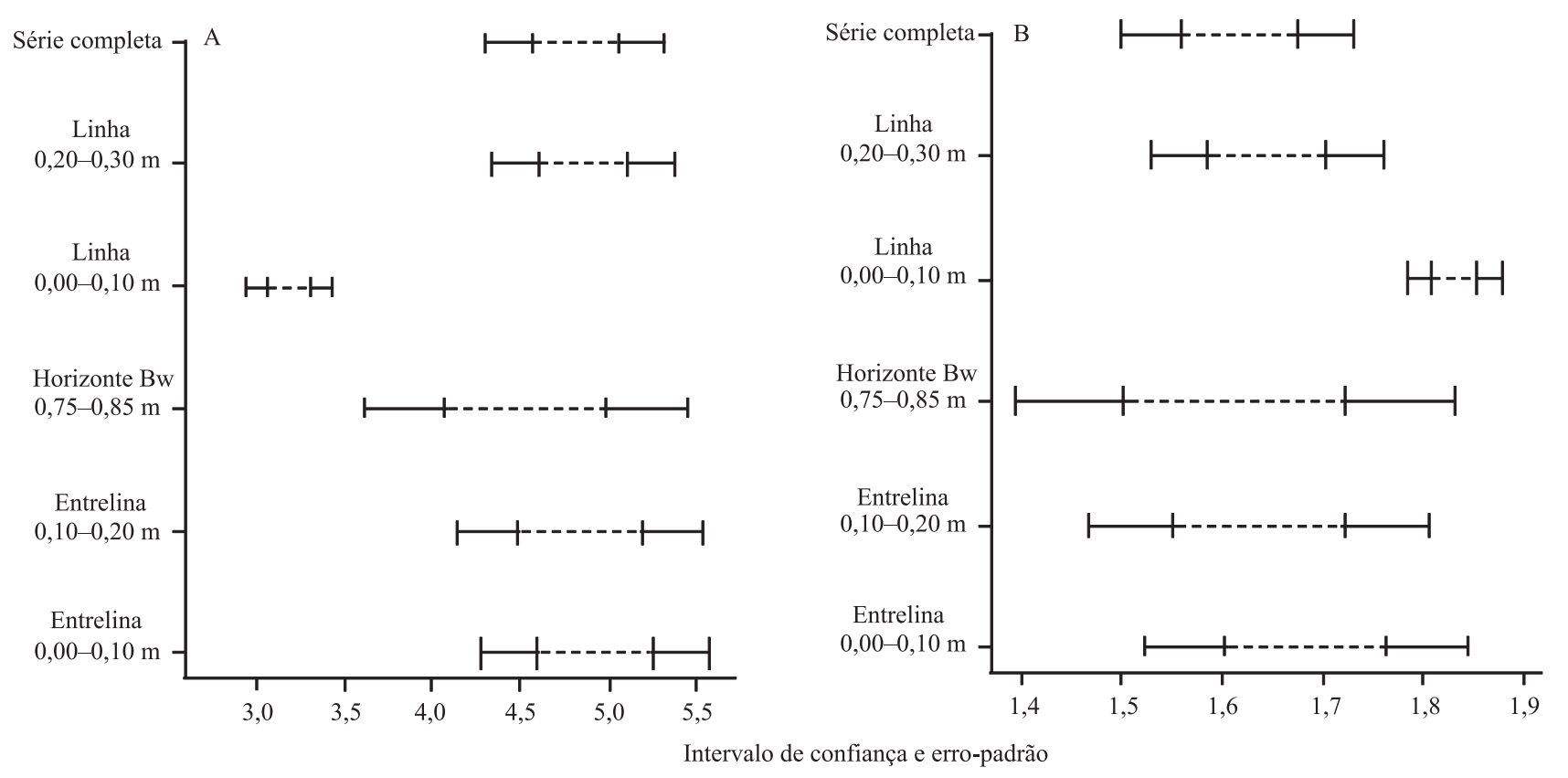

Figura 3. Intervalo de confiança de $95 \%$ (faixa contínua) e erro-padrão (faixa pontilhada) para o coeficiente angular e o intercepto das equações de calibração obtidas por regressão linear. A, intervalo de confiança (95\%) e erro-padrão para o coeficiente angular; B, intervalo de confiança (95\%) e erro-padrão para o intercepto.

teor de água, há maior relação água-ar na condição de maior densidade. Além disso, como a constante dielétrica do ar é próxima de 1 e a da água pode chegar a 80 , haveria elevação da constante dielétrica aparente do meio.

\section{Conclusões}

1. As calibrações mineral e orgânica recomendadas pelo fabricante não se mostraram adequadas, nas condições de manejo avaliadas. 
2. Na inviabilidade de averiguar a acurácia obtida pelo método recomendado pelo fabricante, o uso de ajustes de regressão linear ou da ferramenta Solver mostra-se útil no processo de calibração.

3. São necessárias apenas duas equações de calibração para as situações contrastantes de manejo avaliadas.

4. O manejo do solo afeta a estimativa da umidade do solo por meio da alteração na densidade do solo e em seu teor de matéria orgânica.

\section{Agradecimentos}

Ao Conselho Nacional de Desenvolvimento Científico e Tecnológico, por concessão de bolsa; à Empresa de Pesquisa Agropecuária de Minas Gerais e à Empresa Agropecuária Piumhi, pelo apoio.

\section{Referências}

BAUMHARDT, R.L.; LASCANO, R.J.; EVETT, S.R. Soil material, temperature, and salinity effects on calibration of multisensor capacitance probes. Soil Science Society of America Journal, v.64, p.1940-1946, 2000.

CAMARGO, A.P. de; SENTELHAS, P.C. Avaliação do desempenho de diferentes métodos de estimativa da evapotranspiração potencial no Estado de São Paulo, Brasil. Revista Brasileira de Agrometeorologia, v.5, p.89-97, 1997.

COELHO, E.F.; ANDRADE, C. de L.T.; OR, D.; LOPES, L.C.; SOUZA, C.F. Desempenho de diferentes guias de ondas para uso com o analisador de umidade TRASE. Revista Brasileira de Engenharia Agrícola e Ambiental, v.5, p.81-87, 2001.

DEAN, T.J.; BELL, J.P.; BATY, A.J.B. Soil moisture measurement by an improved capacitance technique. 1. Sensor design and performance. Journal of Hydrology, v.93, p.67-78, 1987.

DELTA-T DEVICES. Profile probe type PR2: user manual. Version 1.2. Cambridge: Delta-T Devices, 2001.

DELTA-T DEVICES. ThetaProbe soil moisture sensor - ML2x: user manual. Cambridge: Delta-T Devices, 1999.

EVETT, S.R.; TOLK, J.A.; HOWELL, T.A. Soil profile water content determination: sensor accuracy, axial response, calibration, temperature dependence, and precision. Vadose Zone Journal, v.5, p.894-907, 2006.

FARES, A.; HAMDHANI, H.; POLYAKOV, V.; DOGAN, A.; VALENZUELA, H. Real-time soil water monitoring for optimum water management. Journal of the American Water Resources Association, v.42, p.1527-1535, 2006.

GUERRA, A.F.; ROCHA, O.C.; RODRIGUES, G.C.; SANZONOWICZ, C.; SAMPAIO, J.B.R.; SILVA, H.C.; ARAÚJO, M.C. de. Irrigação do cafeeiro no cerrado: estratégia de manejo de água para uniformização de florada.
Planaltina: Embrapa Cerrados, 2005. 4p. (Embrapa Cerrados. Comunicado técnico, 122).

HUANG, Q.; AKINREMI, O.O.; RAJAN, R.S.; BULLOCK, P. Laboratory and field evaluation of five soil water sensors. Canadian Journal of Soil Science, v.84, p.431-438, 2004.

KAISER, D.R.; REINERT, D.J.; REICHERT, J.M.; MINELLA, J.P.G. Dielectric constant obtained from TDR and volumetric moisture of soils in southern Brazil. Revista Brasileira de Ciência do Solo, v.34, p.649-658, 2010.

LUKANU, G.; SAVAGE, M.J. Calibration of a frequency-domain reflectometer for determining soil-water content in a clay loam soil. Water SA, v.32, p.37-42, 2006.

MALICKI, M.A.; PLAGGE, R.; ROTH, C.H. Improving the calibration of dielectric TDR soil moisture determination taking into account the solid soil. European Journal of Soil Sciences, v.47, p.357-366, 1996.

NIJLAND, W.; MEIJDE, M. van der; ADDINK, E.A.; JONG, S.M. de. Detection of soil moisture and vegetation water abstraction in a Mediterranean natural area using electrical resistivity tomography. Catena, v.81, p.209-216, 2010.

OELMANN, Y.; KREUTZIGER, Y.; TEMPERTON, V.M.; BUCHMANN, N.; ROSCHER, C.; SCHUMACHER, J.; SCHULZE, E.D.; WEISSER, W.W.; WILCKE, W. Nitrogen and phosphorus budgets in experimental grasslands of variable diversity. Journal of Environmental Quality, v.36, p.396-407, 2007.

PALTINEANU, I.C.; STARR, J.L. Real-time soil water dynamics using multisensor capacitance probes: laboratory calibration. Soil Science Society of America Journal, v.61, p.1576-1585, 1997.

POLYAKOV, V.; FARES, A.; RYDER, M.H. Calibration of a capacitance system for measuring water content of tropical soil. Vadose Zone Journal, v.4, p.1004-1010, 2005.

PREVEDELLO, C.L.; MAGGIOTTO, S.R.; LOYOLA, J.M.T.; DIAS, N.L.; BEPPLER NETO, G. Balanço de água por aquisição automática de dados em cultura de trigo (Triticum aestivum L.). Revista Brasileira de Ciência do Solo, v.31, p.1-8, 2007.

SÁ, M.A.C. de; SANTOS JUNIOR, J. de D.G. dos; SANO, E.E. Calibração e validação de sensor dielétrico para estimativa de teor de água em solos do Cerrado. Planaltina: Embrapa Cerrados, 2008. 32p. (Embrapa Cerrados. Comunicado técnico, 223).

SANTOS, M.R. dos; ZONTA, J.H.; MARTINEZ, M.A. Influência do tipo de amostragem na constante dielétrica do solo e na calibração de sondas de TDR. Revista Brasileira de Ciência do Solo, v.34, p.299-307, 2010.

SILVA, C.R. da; ANDRADE JÚNIOR, A.S. de; ALVES JÚNIOR, J. Calibration of a capacitance probe in a Paleudult. Scientia Agricola, v.64, p.636-640, 2007.

SILVA, E.L. da; GERVÁSIO, E.S. Uso do instrumento TDR para determinação do teor de água em diferentes camadas de um Latossolo Roxo distrófico. Revista Brasileira de Engenharia Agrícola e Ambiental, v.3, p.417-420, 1999. 
STACHEDER, M.; KOENIGER, F.; SCHUHMANN, R. New dielectric sensors and sensing techniques for soil and snow moisture measurements. Sensors, v.9, p.2951-2967, 2009.

STARR, J.L.; PALTINEANU, I.C. Real-time soil water dynamics over large areas using multisensor capacitance probes and monitoring system. Soil and Tillage Research, v.47, p.43-49, 1998.

TOMMASELLI, J.T.G.; BACCHI, O.O.S. Calibração de um equipamento de TDR para medida da umidade de solos. Pesquisa Agropecuária Brasileira, v. 36, p.1145-1154, 2001.

TOPP, G.C.; DAVIS, J.L.; ANNAN, A.P. Electromagnetic determination of soil water content: measurements in coaxial transmission lines. Water Resources Research, v.16, p.574-582, 1980.

VELDKAMP, E.; O'BRIEN, J.J. Calibration of a frequency domain reflectometry sensor for humid tropical soils of volcanic origin. Soil Science Society of America Journal, v.64, p.1549-1553, 2000.

VILLWOCK, R.; TAVARES, M.H.F.; VILAS BOAS, M.A. Calibração de um equipamento TDR em condições de campo. Irriga, v.9, p.82-88, 2004.

WILlmotT, C.J. On the validation of models. Physical Geography, v.2, p.184-194, 1981.

Recebido em 27 de julho de 2011 e aprovado em 30 de janeiro de 2012 